\title{
Effect of Heat on the Circular Dichroism of Spectrin in Hereditary Pyropoikilocytosis
}

\author{
Katherine Chang, Joseph R. Williamson, and Harold S. Zarkowsky, \\ Departments of Pathology and Pediatrics, Washington University School of \\ Medicine and St. Louis Children's Hospital, St. Louis, Missouri 63110
}

\begin{abstract}
A B S T RACT Hereditary pyropoikilocytosis is a hemolytic anemia in which the erythrocytes show increased sensitivity to heat-induced fragmentation. Circular dichroism measurements were employed to study the effect of heat on the secondary structure of pyropoikilocyte membrane proteins. The magnitude of the ellipticity at $222 \mathrm{~nm}$ over the temperature range from $25^{\circ}$ to $70^{\circ} \mathrm{C}$ was determined for erythrocyte ghosts, spectrin, and ghost residue after extraction. In pyropoikilocyte ghosts, protein denaturation began at a lower temperature and the midpoint of the structural transition was displaced from $49^{\circ} \mathrm{C}$ (the value for normal ghosts) to $44^{\circ} \mathrm{C}$. This thermal transition was present in spectrin, but not in the ghost residue after extraction. We conclude that an abnormality in the spectrin molecule alters the physical and morphologic properties of the erythrocyte membrane in pyropoikilocytosis.
\end{abstract}

\section{INTRODUCTION}

Sensitivity to heat-induced morphologic changes was used by Zarkowsky et al. to characterize the erythrocytes of a congenital hemolytic anemia, that they designated pyropoikilocytosis $(1,2)$. For normal erythrocytes the critical temperature of fragmentation is $49^{\circ}$ to $50^{\circ} \mathrm{C}$, whereas pyropoikilocytes were observed to fragment at $45^{\circ} \mathrm{C}$, a temperature at which normal erythrocytes show no morphologic alteration. The erythrocyte morphology in pyropoikilocytosis was also distinctive because of the presence of extremely small spherocytes and cells with bud-like projections.

Earlier studies on the membranes of pyropoikilocytes showed a normal polypeptide pattern on polyacrylamide gel electrophoresis and normal lipid composition (2). We now present evidence that there is an abnor-

This work was presented at the 21st Annual Meeting of the American Society of Hematology and published as an abstract. 1978. Blood. 52(Suppl 1): 62.

Received for publication 14 March 1979 and in revised form 4 April 1979. mality in the spectrin component of the erythrocyte membrane in pyropoikilocytosis.

\section{METHODS}

Whole blood from normal individuals and two siblings with pyropoikilocytosis (2) was collected in vacutainer tubes containing $3.8 \%$ sodium citrate. Erythrocyte ghosts were prepared by the method of Dodge et al. (3). The spectrin extract was prepared by incubating ghosts in $10 \mathrm{vol}$ of $0.1 \mathrm{mM}$ sodium EDTA, pH 8.0 for $15 \mathrm{~min}$ at $37^{\circ} \mathrm{C}$. The supernate and ghost pellet were separated by centrifugation $(37,000 \mathrm{~g}$ for $30 \mathrm{~min})$. The extraction was repeated twice and the pooled supernates were concentrated by pressure filtration using a XM-100A membrane (Amicon Corp., Lexington, Mass.). Thus, three preparations were available from each blood sample: ghosts, extracted and concentrated spectrin, and ghost residue after extraction. Sodium dodecyl sulfate polyacrylamide gel electrophoresis (4) was used to confirm the identity of the preparations (Fig. 1).

Circular dichroism measurements were made in a J-20 spectropolarimeter (Japan Spectrophotometer Co., Tokyo, Japan) using a thermostated optical cell of $10 \mathrm{~mm}$ path-length. The measurements of circular dichroism were performed at $222 \mathrm{~nm}$, the wavelength at which erythrocyte ghosts and spectrin show maximum negative ellipticity (5). All samples were in 77 mosM sodium phosphate buffer, pH 7.4, and kept at the desired temperature for $15 \mathrm{~min}$ before measurements were taken. Measurements were performed at temperature intervals of $1^{\circ}-2^{\circ} \mathrm{C}$ between $20^{\circ}$ and $70^{\circ} \mathrm{C}$. To determine the reversibility of the heat-induced changes in circular dichroism, measurements were performed on samples heated to $40^{\circ}, 50^{\circ}$, and $70^{\circ} \mathrm{C}$ and then repeated after cooling to $25^{\circ} \mathrm{C}$. Data were reduced to obtain the molar ellipticity $([\theta])(5)$. Protein concentration was determined by the method of Lowry et al. (6) with bovine serum albumin as standard.

\section{RESULTS}

The effect of increasing temperature on the circular dichroism of ghosts and spectrin was a decrease in the magnitude of the ellipticity. The effect was irreversible, because the decreased ellipticity was still observed after heated samples had been cooled. The addition of $0.5 \mathrm{mM}$ dithiothreitol had no effect on heat-induced changes in circular dichroism spectra. 


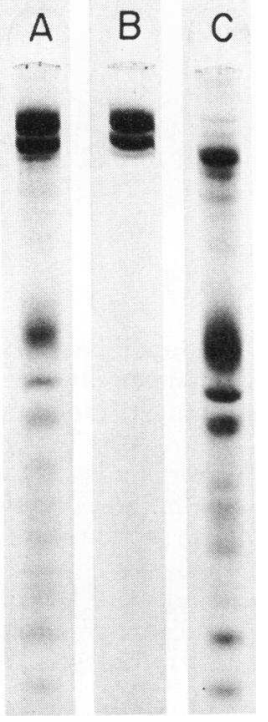

Figure 1 Sodium dodecyl sulfate polyacrylamide gels of membrane preparations from normal erythrocytes. Spectrin was prepared by incubating ghosts in $0.1 \mathrm{mM}$ sodium EDTA, and then purifying and concentrating the extract by pressure filtration. (A) Ghosts. (B) Spectrin. (C) Ghost residue after extraction.

To compare the spectra of the normal and pyropoikilocyte preparations, the data were expressed as the thermal variation parameter, $\Delta[\theta] / \Delta \mathrm{T}$, which is plotted as a function of the temperature (T). In Fig. 2 are shown the results of the circular dichroism studies on ghosts. There are the two transitions, that Brandts et al. (7) designated $\mathrm{A}$ and $\mathrm{B}$. The $\mathrm{A}$ transition is the major one, beginning at $40^{\circ} \mathrm{C}$ with a midpoint at $49^{\circ} \mathrm{C}$. The smaller $B$ transition is seen as a shoulder near $55^{\circ} \mathrm{C}$. Both transitions were observed in pyropoikilocyte ghosts. However, the A transition is shifted to lower temperatures, the midpoint being displaced to $44^{\circ} \mathrm{C}$.

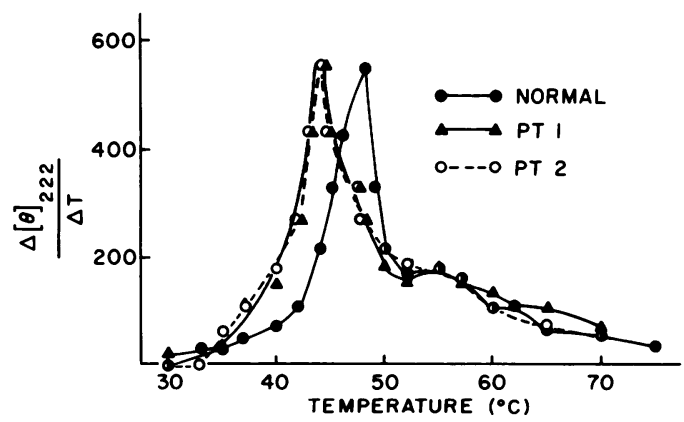

Figure 2 Effect of temperature on circular dichroism of ghosts. Circular dichroism measurements were performed at $222 \mathrm{~nm}$. The thermal variation parameter $(\Delta[\theta] / \Delta \mathrm{T})$ was calculated from differences in the molar ellipticity over temperature $(\mathrm{T})$ intervals of $1^{\circ}-2^{\circ} \mathrm{C}$. Ghosts were prepared from normal erythrocytes and erythrocytes from two patients with pyropoikilocytosis (PT 1 and PT 2).

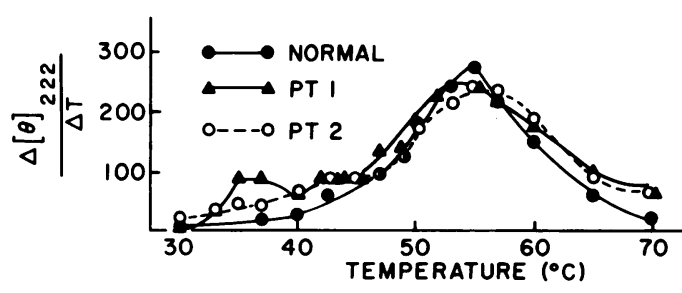

FIGURE 3 Effect of temperature (T) on circular dichroism of ghost residue after extraction. PT, patient with pyropoikilocytosis.

Ghost residues from normal erythrocytes and pyropoikilocytes (Fig. 3) display only the $\mathrm{B}$ transition, with the midpoint at $55^{\circ} \mathrm{C}$.

Spectrin displays a single transition corresponding to the major transition observed in ghosts (Fig. 4). In pyropoikilocyte spectrin, the temperature-dependent change in circular dichroism was again shifted and the peak change in ellipticity occurred at $44^{\circ} \mathrm{C}$ compared to $49^{\circ} \mathrm{C}$ for normal spectrin.

\section{DISCUSSION}

By using scanning calorimetry and circular dichroism, Brandts et al. identified four structural transitions of erythrocyte ghosts that occurred over the temperature range between $40^{\circ}$ and $80^{\circ} \mathrm{C}$ (7). The first, or $A$ transition, centers at $49^{\circ} \mathrm{C}$. The $\mathrm{A}$ transition is not present in ghosts after low-ionic strength extraction, but solubilized spectrin complex demonstrates this thermal transition. They concluded that the $\mathrm{A}$ transition is a result of protein unfolding of the spectrin molecule.

The results of our studies on normal ghosts and spectrin are similar to those of Brandts et al. However, we observe only the A transition in spectrin, whereas Brandts et al. describe a second, minor transition centered at $60^{\circ} \mathrm{C}$. Their spectrin preparation was obtained

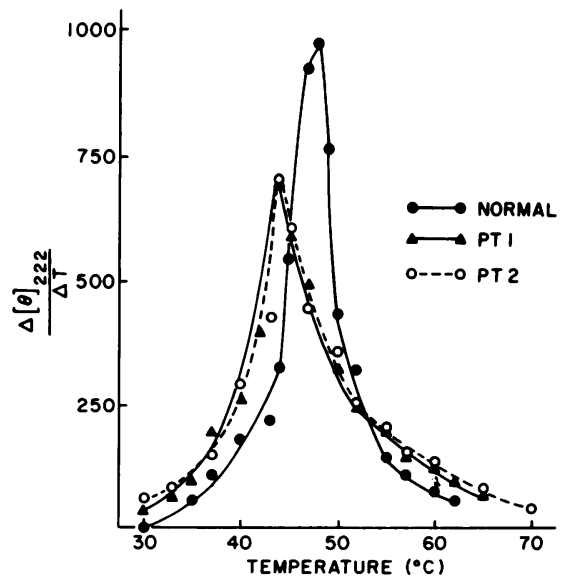

FIGURE 4 Effect of temperature (T) on circular dichroism of spectrin. PT, patient with pyropoikilocytosis. 
after low-ionic strength extraction without further purification. It is likely that the second transition is caused by another membrane component, that was removed during the purification of our preparation.

In these studies circular dichroism was used to monitor the denaturation of membrane protein. The denaturation of spectrin extracted from pyropoikilocyte ghosts progressed at lower temperatures and the peak change in circular dichroism occurred at a temperature $4^{\circ} \mathrm{C}$ lower than that observed for normal spectrin. We suggest that an abnormality in the spectrin molecule accounts for the increased sensitivity to heat denaturation. Coupled with the previously documented heatinduced morphologic changes and in vivo poikilocytosis, the current studies indicate that spectrin is an important determinant of physical properties of the erythrocyte membrane that are relevant under physiologic conditions.

It is interesting that the midpoint of the $\mathrm{A}$ transition corresponds to the critical temperature of heat-induced fragmentation for normal erythrocytes as well as pyropoikilocytes. Brandts et al. also noted that morphologic changes in ghosts occur at a temperature close to the center of the A transition (7). They suggested that the region of spectrin responsible for the A transition played an important role in maintaining membrane structure. This region, however, did not appear to interact directly with the membrane. Our studies strongly support the concept that the portion of the spectrin molecule responsible for the A transition plays a critical role in maintaining the structural integrity of the erythrocyte membrane.

\section{ACKNOWLEDGMENTS}

We are grateful to Dr. Alfred Holtzer, Department of Chemistry, Washington University, St. Louis, Mo., for the use of the spectropolarimeter and his many helpful suggestions.

This work was supported in part by National Institutes of Health grant HL-12839.

\section{REFERENCES}

1. Zarkowsky, H. S., N. Mohandas, C. S. Speaker, and S. B. Shohet. 1973. Congenital hemolytic anemia due to thermal sensitivity of the erythrocyte membrane. Blood. 42: 1019. (Abstr.)

2. Zarkowsky, H. S., N. Mohandas, C. S. Speaker, and S. B. Shohet. 1975. A congenital haemolytic anaemia with thermal sensitivity of the erythrocyte membrane. Br. J. Haematol. 29: 537-543.

3. Dodge, J. T., C. Mitchell, and D. J. Hanahan. 1963. The preparation and chemical characteristics of hemoglobinfree ghosts of human erythrocytes. Arch. Biochem. Biophys. 100: 119-130.

4. Fairbanks, G., T. L. Steck, and D. F. H. Wallach. 1971. Electrophoretic analysis of the major polypeptides of the human erythrocyte membrane. Biochemistry. 10: 26062616.

5. Holzwarth, G., J. Yu, and T. L. Steck. 1976. Heterogeneity in the conformation of different protein fractions from the human erythrocyte membrane. J. Supramol. Struct. 4: 161-168.

6. Lowry, O. H., N. J. Rosebrough, A. L. Farr, and R. J. Randall. 1951. Protein measurement with the Folin phenol reagent. J. Biol. Chem. 193: 265-275.

7. Brandts, J. F., L. Erickson, K. Lysko, A. T. Schwartz, and R. D. Taverna. 1977. Calorimetric studies of the structural transitions of the human erythrocyte membrane. The involvement of spectrin in the A transition. Biochemistry. 16: $3450-3454$. 